\title{
Dynamic analysis of autonomous risk avoidance of amphibious floating bridge based on discrete element method and physical engine
}

\author{
WenbinZhang ${ }^{1, \mathrm{a}^{*}}$, MingxinYe $^{2, \mathrm{~b}}$ \\ ${ }^{1}$ Hubei Key Laboratory of Advanced Technology for Automotive Component, Wuhan University of Technology, Wuhan, China. \\ ${ }^{2}$ School of Automotive Engineering, Wuhan University of Technology, Wuhan, China.
}

\begin{abstract}
In this paper, based on an intelligent floating bridge, by using discrete element methods and physical engines, under the action of certain missile repulsion fields, the force process and motion path in the process of autonomous evasion of missiles are studied. Firstly, the static simulation of missile repulsion fields is carried out by using the polynomial least square surface fitting method. According to the strength of repulsion field at different times and the extrusion force between the pontoons, the kinematic equation of the pontoon is established. The equation is discretised by using a discrete element method, and the kinematic equation is obtained according to the time iteration. Then, motion analysis is carried out by using a physical engine on the basis of equation Analysis. Finally, the position parameters before and after the self-evasion missile of the floating bridge are calculated, and the simulation program is written in MATLAB. The dynamic simulation experiment of the whole evasion missile process is carried out, and the results are satisfactory.
\end{abstract}

\section{Introduction}

As a kind of water transportation channel, as shown in Figure 1, floating bridge is widely used in military battlefields and some special operations because of its simple structure, convenient erection and disassembly, strong mobility and geographical adaptability[1]. Because of its special working conditions, it is necessary to have the strategy to make the intelligent floating bridge evade the incoming missile through reasonable decision-making and maneuver. However, due to the short decision-making and execution time in the process of avoiding missiles, in the face of the enemy's incoming missile, if only relying on human eyes to achieve evasion, it may not be able to make the right decision in time[3].

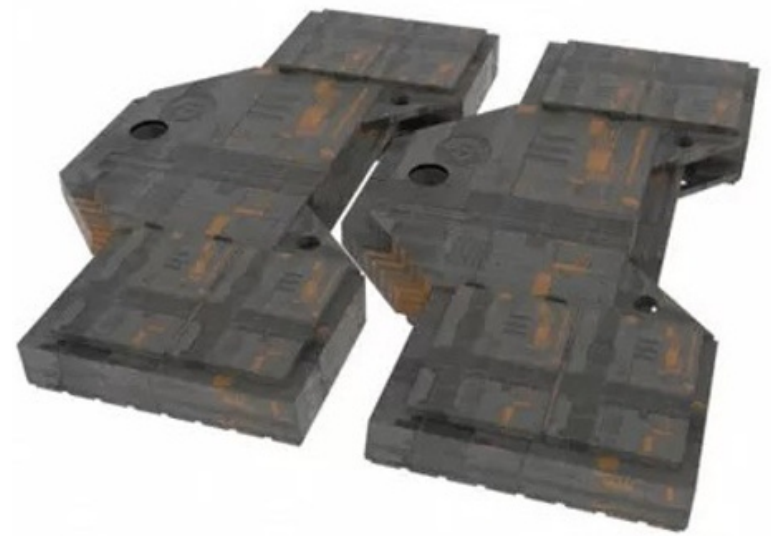

Fig. 1. Schematic diagram of connecting floating bridge.

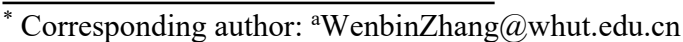

bMingxinYe@whut.edu.cn
}

(c) The Authors, published by EDP Sciences. This is an open access article distributed under the terms of the Creative Commons Attribution License 4.0 


$$
z_{1}+\frac{p_{1}}{\rho g}+\frac{\alpha_{1} v_{1}^{2}}{2 g}=z_{2}+\frac{p_{2}}{\rho g}+\frac{\alpha_{2} v_{2}^{2}}{2 g}+h_{f}
$$

Where $\alpha$ is the kinetic energy correction coefficient and $h_{f}$ is the head loss.

In order to simplify the calculation, the head loss is ignored and the initial state is set to constant. For the pressure distribution in the field,

$$
p+\frac{1}{2} \rho v^{2}=\text { const } .
$$

In order to simulate the real trend of pressure field, the following equations can be established in MATLAB,

$$
\left[\begin{array}{cccc}
R^{3} & R^{2} & R & 1 \\
r_{0}^{3} & r_{0}^{2} & r_{0} & 1 \\
3 R^{2} & 2 R & 1 & 0 \\
3 r_{0}^{2} & 2 r_{0} & 1 & 0
\end{array}\right]\left[\begin{array}{l}
\mathrm{a} \\
b \\
c \\
d
\end{array}\right]=\left[\begin{array}{c}
P_{\min } \\
P_{\max } \\
0 \\
0
\end{array}\right]
$$

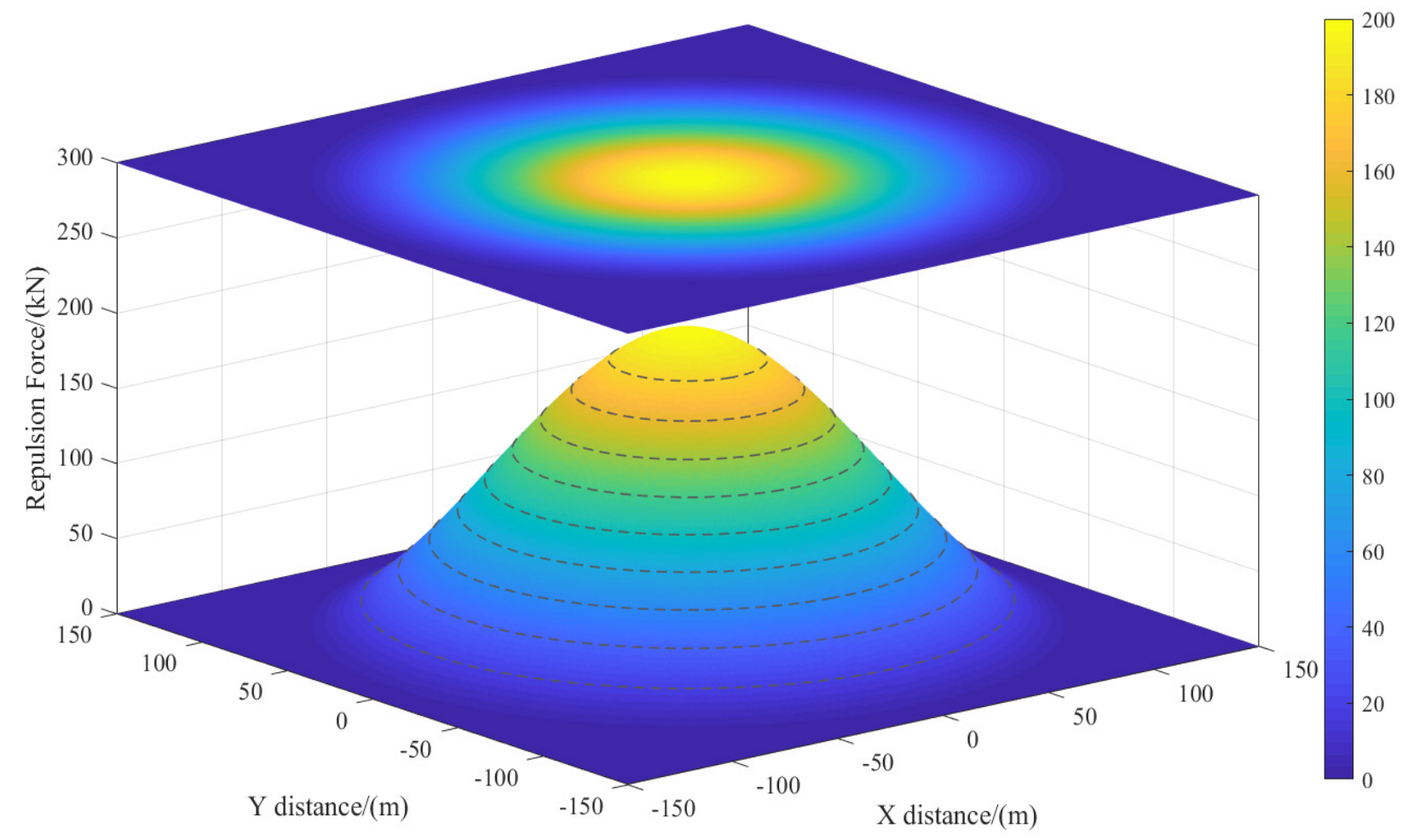

Fig. 2. Generated repulsion field

\subsection{Positioning}

For the bridge body in repulsion field, take it as the research object. Assuming the initial coordinate is $\left(x_{0}\right.$, $y_{0}$ ), and the equivalent force area is $S$, the repulsion force is:

$$
F(x, y)=S \cdot P(x, y)
$$

Where Pmax is the maximum relative pressure at the center of the field and Pmin is the minimum relative pressure at the edge of the field, where is 0 .

Given different maximum and minimum relative pressures, the corresponding coefficients obtained are shown in the table below.

Table 1. The values of parameters under different relative pressures.

\begin{tabular}{|c|c|c|c|c|}
\hline$P_{\max }$ & 200 & 200 & 300 & 300 \\
\hline$P_{\min }$ & 0 & 10 & 0 & 20 \\
\hline $\mathrm{a}$ & $1.19 \mathrm{e}-04$ & $1.13 \mathrm{e}-04$ & $1.78 \mathrm{e}-04$ & $-1.66 \mathrm{e}-04$ \\
\hline $\mathrm{b}$ & -0.0267 & -0.0253 & -0.0400 & -0.0373 \\
\hline $\mathrm{c}$ & 0 & 0 & 0 & 0 \\
\hline $\mathrm{d}$ & 200 & 200 & 300 & 300 \\
\hline
\end{tabular}

Therefore, for any point in the field, it is satisfied

$$
\mathrm{p}(x, y)=\operatorname{polyval}\left(p_{0}, x^{2}+y^{2}\right)
$$

Where, $P_{0}=[a, b, c, d]$.

For the first group of data, the force field generated is shown in Figure 2.

For the centre position of the field, $P(x, y)=P_{\max }$, then $F(x, y)=F_{\max }$, Then the above formula can be expressed as:

$$
F_{p}=\frac{F_{\max }}{\left(x_{0}^{2}+y_{0}^{2}\right)}
$$

The azimuth of the pressure is as follows: 


$$
\theta_{F_{p}}=\arctan \left(\frac{y_{0}}{x_{0}}\right)
$$

The resulting acceleration is:

$$
a=\frac{F_{p}}{m}
$$

The velocity and position coordinates are discretized, and the initial velocity of a single floating bridge is set as $v_{0}$, and:

$$
v_{0}=0
$$

In the $d t$-th time,

$$
d v=a \cdot d t=\frac{F_{\max }}{\left(x_{t}^{2}+y_{t}^{2}\right) m} \cdot d t
$$

Therefore:

$$
v_{t}=\int_{0}^{t} \frac{F_{\max }}{\left(x_{t}^{2}+y_{t}^{2}\right) m} \cdot d t
$$

So we can get the new coordinates in the next time:

$$
\begin{gathered}
d x=v_{t} \cos \theta_{F_{p}} \cdot d t \\
d y=v_{t} \sin \theta_{F_{p}} \cdot d t
\end{gathered}
$$

By integrating the above formula, the coordinates of the floating bridge at any time $t$ can be obtained.

\section{Simulation and analysis of floating bridge's autonomous risk avoidance motion based on physical engine}

In order to carry out the simulation test in the computer, the above formula needs to be further discretized. For the $i$-th moment, the magnitude of the field force on the floating bridge is as follows:

$$
F(i)=\frac{F_{\max }}{x_{0}^{2}(i)+y_{0}^{2}(i)}
$$

The azimuth is as follows:

$$
\theta(i)=\arctan \left(\frac{y_{0}(i)}{x_{0}(i)}\right)
$$

Acceleration at current time:

$$
a(i)=\frac{F(i)}{m}
$$

Get the speed of the next moment:

$$
v(i+1)-v(i)=a(i) \cdot d t
$$

Then the coordinate position of the next time is obtained:

$$
\begin{gathered}
x(i+1)-x(i)=v(i) \cos (\theta(i)) \cdot d t \\
y(i+1)-y(i)=v(i) \sin (\theta(i)) \cdot d t
\end{gathered}
$$

Put the above formula into Matlab and set a specific time step for iteration. For the initial state, the force of a single floating bridge is shown in Figure 3, where $F_{l}$ is the repulsive force generated in the repulsive field, and
$F_{2}$ is the total force generated by the extrusion between the bridge and the girder. The resultant force of the two forces can cause the horizontal displacement of the girder.

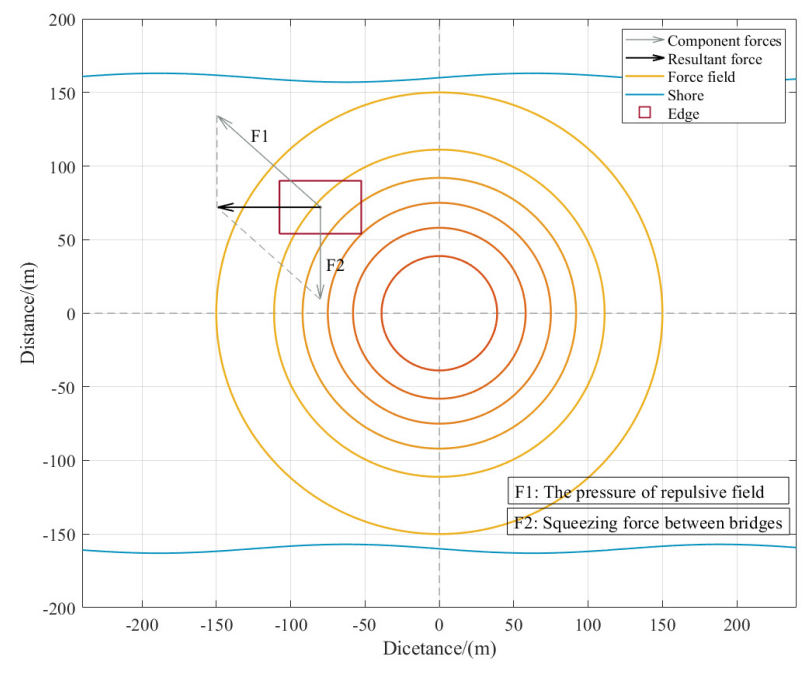

Fig. 3. Initial stress analysis of single floating bridge

Because the repulsion force in the field will decrease with the increase of the distance from the centre of the field, the total squeezing force between the bridges will also decrease adaptively, and the gravity toward the centre will increase with the increase of the displacement distance, so there is a steady point for each beam segment, which makes the force of the floating bridge tend to be stable. The final position of the floating bridge in the force field is shown in Figure 4.

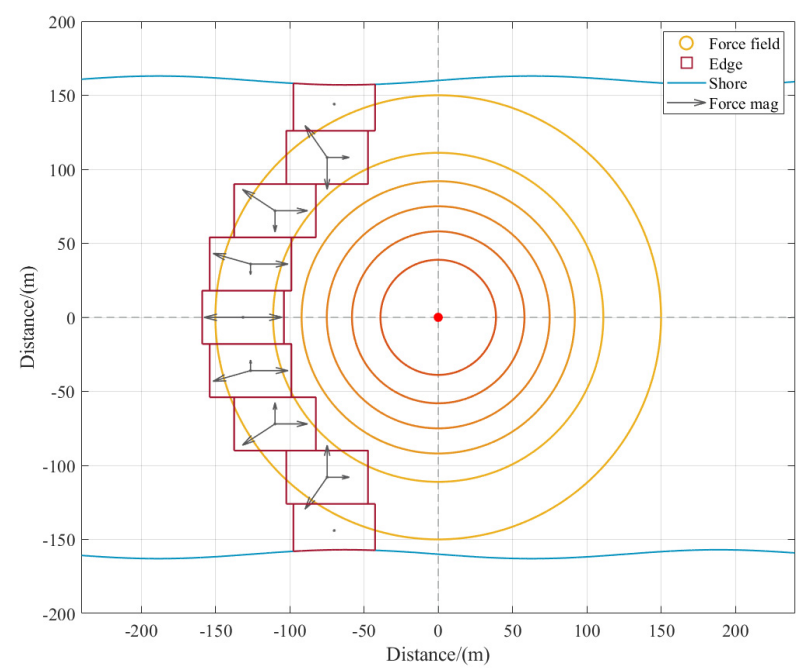

Fig. 4. The position and stress state of the floating bridge in the final state of motion

After evading the missile, the repulsion field disappears. At the same time, the squeezing force between the pontoons caused by the repulsive force field also disappears synchronously, and the pontoon will start to move again under the action of its original position gravity. This is because after the repulsion field is removed, the repulsion from the field disappears, and the squeezing force from other pontoons also disappears. For a single pontoon, it is attracted to the original position, 
and gradually returns to its original position, reaching the stable connection state again.

The process is simulated in the physical engine built by MATLAB. After the repulsion field is removed, the simulation results and forces of the initial state and final state of the floating bridge are respectively shown in Figure 5. The floating bridge gradually returns to the initial position under the action of horizontal right attraction. With the decrease of the distance between the floating bridge and the initial position, the attraction received by the floating bridge is smaller, and finally it can reach the straight-line connection state again, as shown in Figure 6.

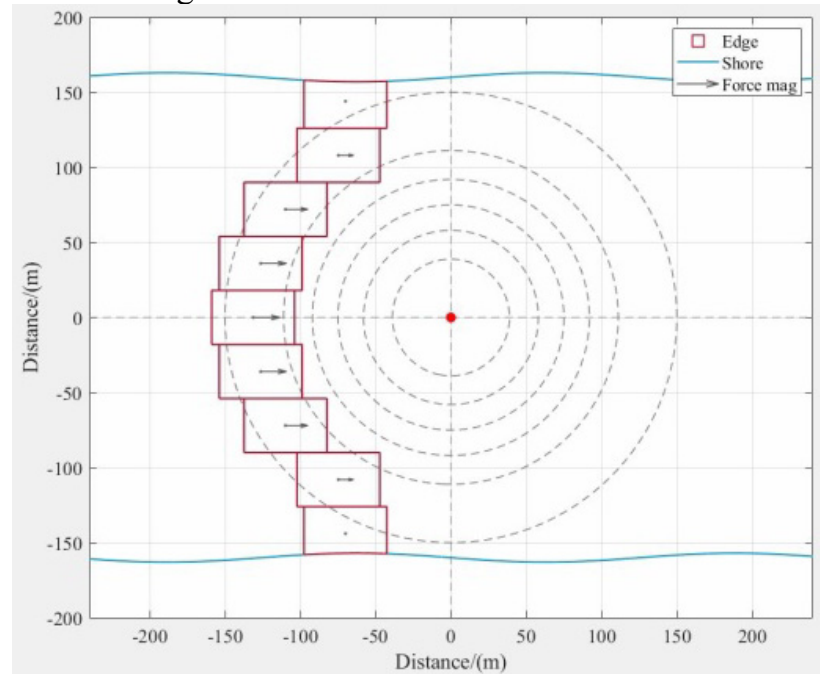

Fig. 5. Simulation results of initial state after the repulsion field disappears

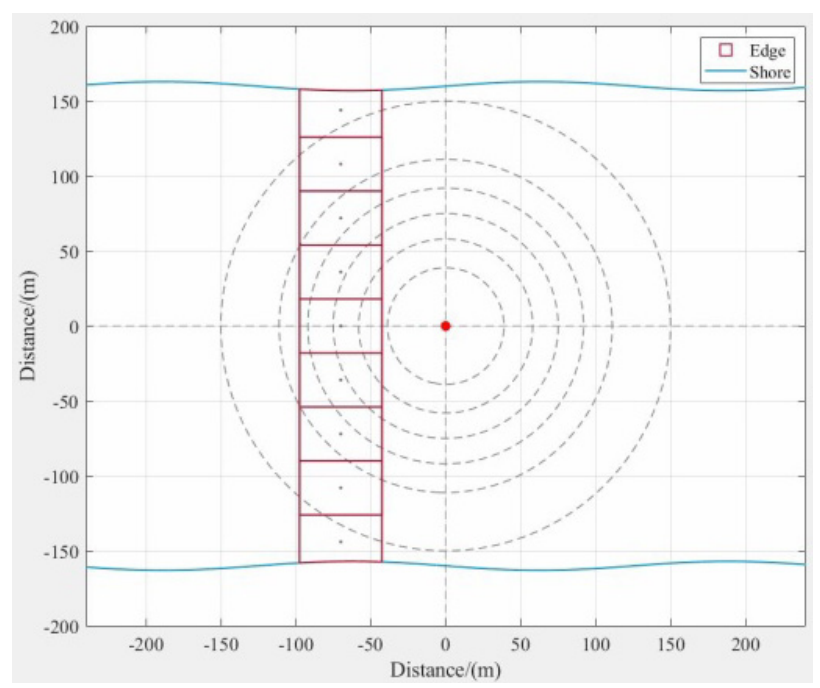

Fig.6. Simulation results of the final state after the repulsion field disappears

It can be seen that the recovery effect of the floating bridge after avoiding the missile is good, and the simulation results are consistent with the actual application results.

\section{Conclusions}

In this paper, based on a military intelligent floating bridge, the impact repulsion field of a missile is simulated statically by using polynomial least square surface fitting method, and the motion of the missile under the repulsion field is analysed by using discrete element method and autonomous physical engine, and the strategy of avoiding missile is studied. Finally, the modelling and simulation are carried out in MATLAB, and the simulation results are compared It is satisfactory and helpful for the manoeuvrability and safety of the intelligent floating bridge.

\section{Acknowledgments}

This work was supported by the National College Students' energy saving and emission reduction and social practice competition project [414-3120600201].

\section{References}

1. Miao, Y., Chen, X., Ye, Y. (2021) Research on dynamic performance of floating bridges subjected to wave, current and moving loads. [J]. Journal of Ship Mechanics,2021,25(2):228-237.

2. Wang, X., Wang, X., Dong, Y. (2020) Visual simulation for structure continuous collapse based on discrete element method and physical engine. [J]. Journal of Vibration and Shock, 2020,39(13):267275.

3. Zhou, J. Analysis and Simulation of dynamic characteristics of aircraft missile ejection system. Shanxi: Northwest University of Technology, 2004.

4. Fan, X., Li, D., Zhang, W. Missile Evasion Decision Training Based on Deep Reinforcement Learning[J]. Electronics Optics \& Control, 2021, 28(1):81-85.

5. Shao, Y., Zhu, R., He, Jl. Exploration of A New Evasive Maneuver Mode for Medium and Long Range Air-to-air Radar Missile[J]. Journal of Projectiles, Rockets, Missiles and Guidance, 2020, 40(4):75-78,84.

6. Fumiaki I, Takeshi K. A method to solve missileaircraft pursuit-evasion differential games[J], IFAC Proceedings Volumes, 2005,38,(1), 176-181.

7. Carr, R., Torf, J., Cobb, R. Cooperative Control for Missile Evasion[C]. AIAA Guidance, Navigation, \& Control Conference. 2015.

8. Zhu, Q., Shao, Z. Real-time receding horizon pursuit and evasion games of missile guidance based on neural network[J]. Systems Engineering and Electronics, 2019,41(7):1597-1605.

9. Thomas, J., James, D. Yu, X. Aerodynamics of small vehicles[J]. Advances in mechanics,2004, 34(2):270-279. 